accuracy is available (perhaps from a few iterates of the power method); second, when an over-estimate is preferred to an under-estimate; and third, when no further improvement of the eigenvector is contemplated. These conditions are found in the problem of computing the optimum successive over-relaxation parameter.

No cases have been found for which $\sigma$ gives a faster convergence rate than $\mu(\alpha)$ for any reasonable value of $\alpha$. Dangers of many kinds exist for slowly convergent problems but this particular one has not been observed. Furthermore, any increase in convergence rate gained by use of this method reduces the likelihood that the sequence $(2 \mathrm{a}, 2 \mathrm{~b})$ and (3) will be terminated by a false convergence indication.

Bettis Atomic Power Laboratory*

Pittsburgh, Pennsylvania

1. S. H. Crandall, Engineering Analysis, A Survey of Numerical Procedures, McGraw-Hill, New York, 1956. MR 18, 674.

2. G. H. GolUB \& R. S. VARGA, "Chebyshev semi-iterative methods, successive overrelaxation methods, and second order Richardson iterative methods. I, II," Numer. Math., v. 3, 1961, pp. 147-168. MR 26 *3207.

3. D. S. GRIfFIn \& R. S. VARGA, "Numerical solution of plane elasticity problems," $J$. Soc. Indust. Appl. Math., v. 11, 1963, pp. 1046-1062. MR 28 *3544.

4. R. S. VARGA, On Estimating Rates of Convergence in Multigroup Diffusion Problems, WAPD-TM-41, OTS, U. S. Dept. of Commerce, Washington, D. C., 1957.

5. R. S. VARga, Matrix Iterative Analysis, Prentice-Hall, Englewood Cliffs, N. J., 1962. MR $28 * 1725$.

* Now at Westinghouse Research and Development Center, Pittsburgh, Pennsylvania.

\title{
A Practical Application of Block Diagonally Dominant Matrices
}

\section{By H. S. Price}

Introduction. In this note the concept of block diagonally dominant matrices (see references [3], [4], [5]) is applied to a problem from electromagnetic theory. The actual problem considered here is to find the vector potential $P(r, z)$, induced in a piecewise homogeneous, axially symmetric, infinite region $\Omega$, by a current loop of radius $r_{t}$ located at $z=0$. Using Maxwell's first two equations (see references [6], [7]), it can be shown that $P(r, z)$ satisfies the following differential equation:

$$
\frac{1}{r} \frac{\partial}{\partial r}\left(r \frac{\partial P(r, z)}{\partial r}\right)+\frac{\partial^{2} P(r, z)}{\partial z^{2}}+\left(k^{2}(r, z)-\frac{1}{r^{2}}\right) P(r, z)=0, \quad(r, z) \in \Omega .
$$

The complex-valued function $k^{2}(r, z)$ is given by

$$
k^{2}=\epsilon(r, z) \mu(r, z) \omega^{2}-i \mu(r, z) \sigma(r, z) \omega=\alpha(r, z)-i \beta(r, z),
$$

where $\mu$ is the permeability, $\sigma$ the conductivity, $\epsilon$ the inductive capacity, and $\omega$ is the angular frequency. At the source, for a current loop of radius $r_{t}$ whose plane is normal to the $z$-axis and whose center is located at the origin, we have 


$$
\nabla^{2} P(r, z)-\frac{1}{r^{2}} P(r, z)+k^{2}(r, z) P(r, z)=-\mu J^{*} \quad\left(r=r_{t} ; z=0\right),
$$

where $J^{*}$ is the current density in the current loop. If we now assume that the source sees the region as homogeneous (which is a fairly good assumption for many practical cases), we can solve equation (3) for $P(r, z)$ along the line $r=r_{t}$, giving

$$
P\left(r_{t}, z\right)=f(z) .
$$

Also, since $P(r, z) \rightarrow 0$ as $r, z \rightarrow \infty$, we can choose a $z_{b}$ and an $r_{b}$ so large that very little error is introduced by assuming

$$
\begin{aligned}
P\left(r_{b}, z\right) & \equiv 0, \\
P\left(r, z_{b}\right) & \equiv 0 .
\end{aligned}
$$

Then equations (4) and (5) along with (1) gives us the following behavior equations,

$$
\begin{gathered}
\frac{1}{r} \frac{\partial}{\partial r}\left(r \frac{\partial P(r, z)}{\partial r}\right)+\frac{\partial^{2} P(r, z)}{\partial z^{2}}+\left(k^{2}(r, z)-\frac{1}{r^{2}}\right) P(r, z)=0, \quad(r, z) \in \Omega, \\
P(r, z)=f(r, z), \quad(r, z) \in \Gamma
\end{gathered}
$$

where $\Gamma$ is the boundary of $\Omega$ and $\Omega$ is defined as all $r$ and $z$ such that

$$
0<r_{t}<r<r_{b} \text { and } 0<z<z_{b} \text {. }
$$

If the behavior equations (6) above are separated into real and imaginary parts and then approximated in the usual way (see [2, pp. 181-187]) the following matrix equation arises,

$$
N \boldsymbol{\phi}=\mathbf{s} .
$$

The $2 n \times 2 n$ matrix $N$ is not diagonally dominant in the usual sense, but we shall prove that it is block diagonally dominant for a certain partitioning.

Difference Approximation. If

$$
P(r, z)=P_{1}(r, z)+i P_{2}(r, z),
$$

we obtain, by separating equation (6a) into real and imaginary parts,

$$
\begin{aligned}
& \text { (10a) } \frac{1}{r} \frac{\partial}{\partial r}\left(r \frac{\partial P_{1}(r, z)}{\partial r}\right)+\frac{\partial^{2} P_{1}(r, z)}{\partial z^{2}}-\left(\frac{1}{r^{2}}-\alpha(r, z)\right) P_{1}(r, z)=-\beta(r, z) P_{2}(r, z), \\
& \text { (10b) } \frac{1}{r} \frac{\partial}{\partial r}\left(r \frac{\partial P_{2}(r, z)}{\partial r}\right)+\frac{\partial^{2} P_{2}(r, z)}{\partial z^{2}}-\left(\frac{1}{r^{2}}-\alpha(r, z)\right) P_{2}(r, z)=\beta(r, z) P_{1}(r, z) .
\end{aligned}
$$

If the $n \times n$ matrix $A=\left(a_{\gamma, \eta}\right)$ is the discrete approximation to the operator

$$
-\nabla^{2}=-\frac{1}{r} \frac{\partial}{\partial r}\left(r \frac{\partial}{\partial r}\right)-\frac{\partial^{2}}{\partial z^{2}}
$$

obtained by integration (see $\left[2\right.$, p. 166]) and $u_{i, j}$ and $v_{i, j}$ are the mesh approximations to $P_{1}\left(r_{i}, z_{j}\right)$ and $P_{2}\left(r_{i}, z_{j}\right)$, respectively, then (10) becomes 


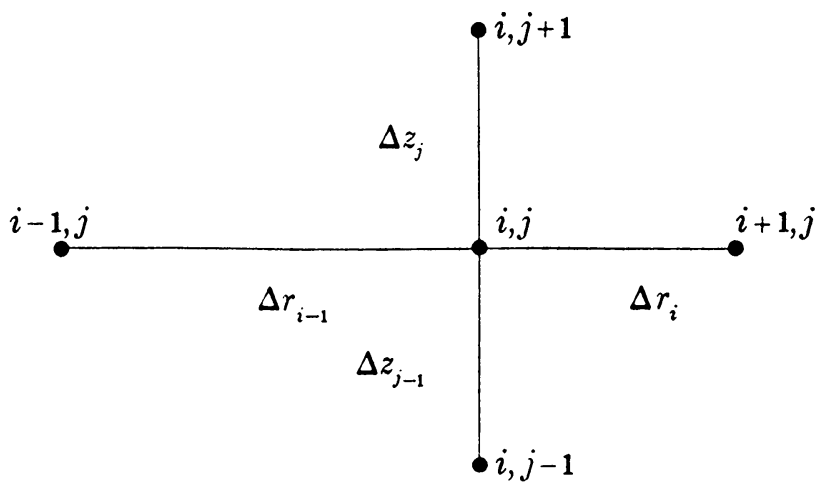

FigURE 1

(12a)

$$
\begin{aligned}
& (A \mathbf{u})_{i, j}+\left(\frac{1}{r_{i}^{2}}-\alpha_{i, j}\right)(E \mathbf{u})_{i, j}=\beta_{i, j}(E \mathbf{v})_{i, j}, \quad 1 \leqq i \leqq I, 1 \leqq j \leqq J, \\
& (A \mathbf{v})_{i, j}+\left(\frac{1}{r_{i}{ }^{2}}-\alpha_{i, j}\right)(E \mathbf{v})_{i, j}=-\beta_{i, j}(E \mathbf{u})_{i, j},
\end{aligned}
$$

where $(A \mathbf{u})_{i, j}$ and $(E \mathbf{u})_{i, j}$ are defined at the $i, j$ th mesh point by

$$
\begin{aligned}
(A \mathbf{u})_{i, j}= & \left(\frac{u_{i, j}-u_{i, j+1}}{\Delta z_{j}}\right)\left(r_{i+1 / 2}^{2}-r_{i-1 / 2}^{2}\right)+\left(\frac{u_{i, j}-u_{i, j-1}}{\Delta z_{j-1}}\right)\left(r_{i+1 / 2}^{2}-r_{i-1 / 2}^{2}\right) \\
& +\left(\frac{u_{i, j}-u_{i+1, j}}{\Delta r_{i}}\right)\left(r_{i+1 / 2}\right)\left(\frac{\Delta z_{j}+\Delta z_{j-1}}{2}\right) \\
& +\left(\frac{u_{i, j}-u_{i-1, j}}{\Delta r_{i-1}}\right)\left(r_{i-1 / 2}\right)\left(\frac{\Delta z_{j}+\Delta z_{j-1}}{2}\right)
\end{aligned}
$$

$(1 \leqq i \leqq I, 1 \leqq j \leqq J)$,

(13b) $(E \mathbf{u})_{i, j}=\left(r_{i+1 / 2}^{2}-r_{i-1 / 2}^{2}\right)\left(\frac{\Delta z_{j}+\Delta z_{j-1}}{2}\right) u_{i, j}$.

(See Figure 1.)

Since with the assumptions stated in the introduction we have just the Dirichlet problem (equation (6b)), equations (12) and (13) are a difference approximation for equations $(6)$.

Block Diagonal Dominance. From now on we will assume that

$$
\alpha_{i, j} \leqq \frac{1}{r_{i}{ }^{2}}, \quad 1 \leqq i \leqq I, 1 \leqq j \leqq J,
$$

since this is the case that leads to an application of block diagonally dominant matrices. This assumption is valid for many interesting physical problems since, for a good conductor (see [7]), $\sigma_{i}^{\prime} \omega \epsilon \gg 1$ and $k$ can be written as

$$
k \approx(1+i)(\omega \mu \sigma)^{1 / 2} .
$$

This implies that $k^{2}$ is pure imaginary or, in other words, $\alpha(r, z) \equiv 0$ in (2).

We now define $D=\left(d_{k, k}\right)$ and $F=\left(f_{k, k}\right)$ to be $n \times n$ diagonal matrices, where 


$$
\begin{aligned}
(D \mathbf{u})_{i, j}=\left(\frac{1}{r_{i}^{2}}-\alpha_{i, j}\right)\left(r_{i+1 / 2}^{2}-r_{i-1 / 2}^{2}\right)\left(\frac{\Delta z_{j}+\Delta z_{j-1}}{2}\right) & u_{i, j} \\
1 & \leqq i \leqq I, 1 \leqq j \leqq J
\end{aligned}
$$

and

$$
(F \mathbf{u})_{i, j}=\beta_{i, j}\left(r_{i+1 / 2}^{2}-r_{i-1 / 2}^{2}\right)\left(\frac{\Delta z_{j}+\Delta z_{j-1}}{2}\right) u_{i, j},
$$

at the $i, j$ th mesh point.

Now, with these definitions, the discrete approximations to (10) in matrix notation become

$$
\begin{aligned}
& A \mathbf{u}+D \mathbf{u}=F \mathbf{v}, \\
& A \mathbf{v}+D \mathbf{v}=-F \mathbf{u}
\end{aligned}
$$

or, defining $M \equiv A+D$, then the matrix equation to be solved is

$$
N \phi=\mathrm{S},
$$

where

$$
N=\left[\begin{array}{cc}
M & -F \\
F & M
\end{array}\right], \quad \phi=\left[\begin{array}{l}
\mathbf{u} \\
\mathbf{v}
\end{array}\right]
$$

and $\mathbf{S}$ is the vector arising from the boundary conditions.

We will now permute the matrix $N$ so that the equation for the real part of $P$, $(u)$, at a given mesh point, is followed by the equation for the imaginary part of $P,(v)$, at the same point. The matrix then has the form:

(20)

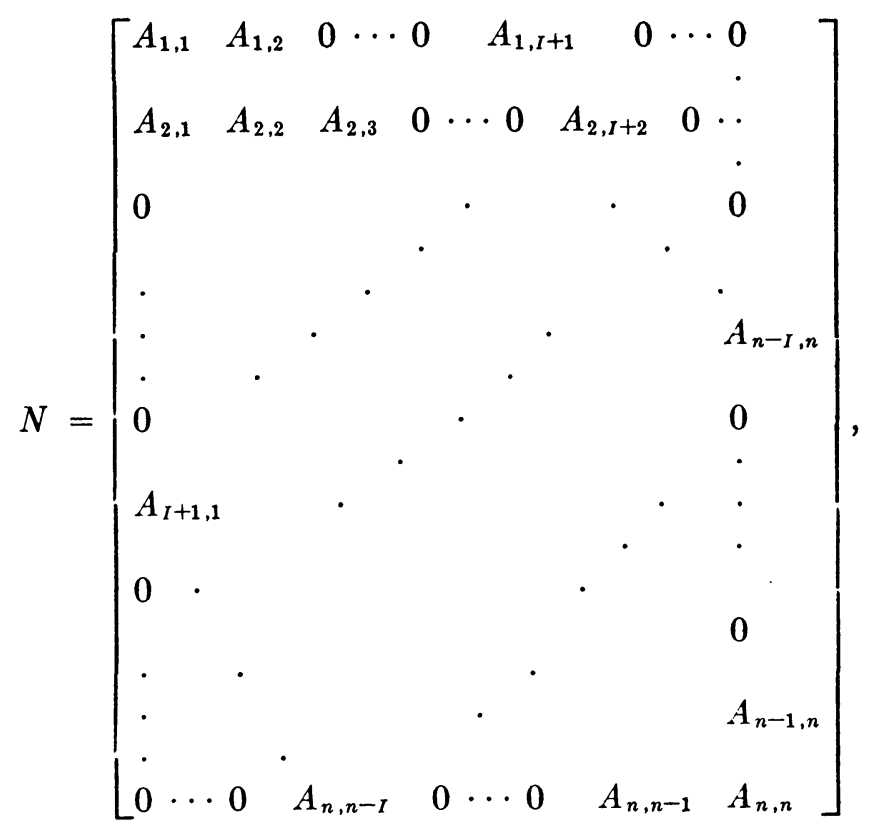

where 


$$
\begin{aligned}
A_{k, k} & =\left[\begin{array}{cc}
\left(a_{k, k}+d_{k, k}\right) & \left(a_{k, k}+d_{k, k}\right) \\
f_{k, k} & -f_{k, k}
\end{array}\right], \\
A_{k, k+1} & =\left[\begin{array}{cc}
-a_{k, k+1} & 0 \\
0 & -a_{k, k+1}
\end{array}\right]=A_{k+1, k}, \text { and } \\
A_{k, I+k} & =\left[\begin{array}{cc}
-a_{k, I+k} & 0 \\
0 & -a_{k, I+k}
\end{array}\right]=A_{I+k, k} .
\end{aligned}
$$

Now, from equation (13a),

$$
\left|a_{k, k}\right| \geqq \sum_{j=1 ; j \neq k}^{n}\left|a_{k, j}\right|
$$

with strict inequality holding for the equations of each point adjacent to the boundary. Notice that if $d_{k, k}=0$ for $1 \leqq k \leqq n$, with certain choices of the $f_{k, k}$ we can have

$$
\left|a_{k, k}\right|<\sum_{j=1 ; j \neq k}^{n}\left|a_{k, j}\right|+\left|f_{k, k}\right|, \quad 1 \leqq k \leqq n,
$$

so $N$ is definitely not diagonally dominant in the usual sense. However, following [3], we define block diagonally dominant matrices as follows:

$$
A=\left[\begin{array}{cccc}
A_{1,1} & A_{1,2} & \cdots & A_{1, n} \\
A_{2,1} & A_{2,2} & \cdots & A_{2, n} \\
\vdots & \vdots & & \vdots \\
A_{n, 1} & A_{n, 2} & \cdots & A_{n, n}
\end{array}\right] .
$$

Definition 1. Let the $2 n \times 2 n$ matrix $A$ be partitioned as in (24). If the diagonal submatrices $A_{j, j}$ are nonsingular, and if

$$
\left(\left\|A_{j, j}^{-1}\right\|\right)^{-1} \geqq \sum_{r=1 ; r \neq j}^{n}\left\|A_{j, r}\right\| \text { for all } 1 \leqq j \leqq n,
$$

where $\|$ \| denotes the matrix norm subordinate to the ordinary Euclidean norm for vectors, then $A$ is block diagonally dominant, relative to the partitioning (24). If strict inequality holds in (25) for $1 \leqq j \leqq n$, then $A$ is block strictly diagonally dominant and if $A$ is block irreducible and strict inequality holds in (25) for at least one $j$ then $A$ is block irreducibly diagonally dominant.

TheOREM 1. The natrix $N$ given in (20) relative to the partitioning shown, is block irreducibly diagonally dominant and consequently is nonsingular.

Proof. It is clear from the form of the $2 \times 2$ matrix $A_{j, j}$ in (21) that

$$
\operatorname{det}\left(A_{j, j}\right)=\left(a_{j, j}+d_{j, j}\right)^{2}+f_{j, j}^{2}
$$

and

$$
\left(\left\|A_{j, j}^{-1}\right\|\right)^{-1}=\left[\operatorname{det}\left(A_{j, j}\right)\right]^{1 / 2}=\left[\left(a_{j, j}+d_{j, j}\right)^{2}+f_{j, j}^{2}\right]^{1 / 2} \geqq\left|a_{j, j}\right| .
$$

The off-diagonal blocks of $N$ are scalar matrices (i.e., diagonal matrices with equal diagonal entries), so

$$
\sum_{i=1 ; i \neq j}^{n}\left\|A_{j, i}\right\|=\sum_{i=1 ; i \neq j}^{n}\left|a_{j, i}\right|
$$




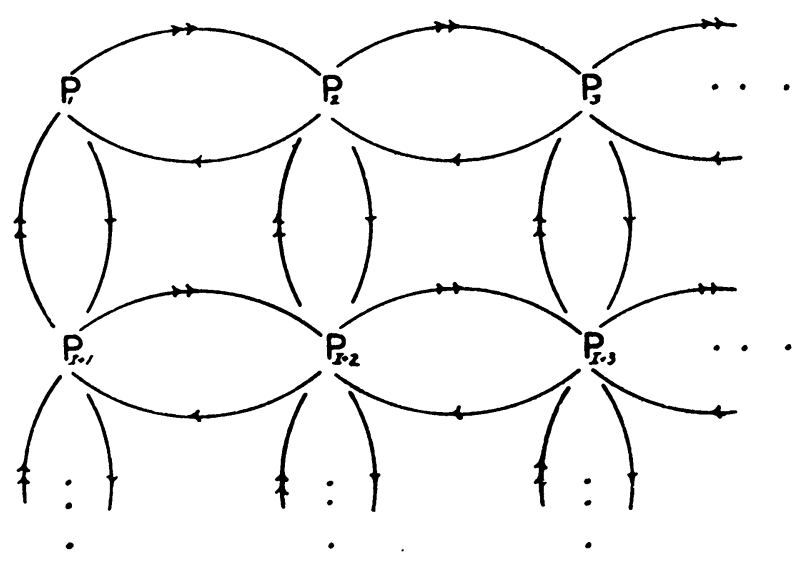

Figure 2

But now, combining equations (22), (26) and (27), we have

$$
\left(\left\|A_{j, j}^{-1}\right\|\right)^{-1} \geqq\left|a_{j, j}\right| \geqq \sum_{i=1 ; i \neq j}^{n}\left|a_{j, i}\right|=\sum_{i=1 ; i \neq j}^{n}\left\|A_{j, i}\right\|,
$$

so from (25) of Definition $1, N$ is block diagonally dominant. Since the block irreducibility follows simply from looking at the block directed graph of $N$ (see Figure $2), N$ is block irreducibly diagonally dominant with strict inequality in (28) for all points adjacent to the boundary. Therefore by Theorem 1 of [3] $N$ is nonsingular.

Lemma $1 . N$ as partitioned in (20) is a consistently ordered 2-cyclic matrix.

Proof. The block directed graph of type $2^{*}$ of the block Jacobi matrix derived from $N$ is given by Figure 2 .

Since every closed path is a multiple of 2 and has equal numbers of major and minor paths (see [2, p. 121]), $N$ is a consistently ordered 2-cyclic matrix.

LemMa 2. The block Jacobi matrix $B$ derived from $N$ is convergent.

Proof. $B=I-D_{1}^{-1} C$, where $N=D_{1}-C$ and $D_{1}$ is a block diagonal matrix with diagonal entries $A_{j, j}$.

Since

$$
\sum_{j=1}^{n}\left\|B_{i, j}\right\| \equiv\left(\sum_{j=1 ; i \neq j}^{n}\left\|A_{i, j}\right\|\right)\left(\left\|A_{i, i}^{-1}\right\|\right)
$$

and, from (28),

$$
\left(\left\|A_{i, i}^{-1}\right\|\right) \leqq\left(\sum_{j=1 ; i \neq j}^{n}\left\|A_{i, j}\right\|\right)^{-1}
$$

we have, finally,

$$
\sum_{j=1}^{n}\left\|B_{i, i}\right\| \leqq 1 \text { for all } i .
$$

Then, from Theorem 2 of [3], if $\lambda_{i}$ is any eigenvalue of $B$,

$$
\left|\lambda_{i}\right| \leqq \sum_{j=1}^{n}\left\|B_{k, j}\right\| \leqq 1
$$

\footnotetext{
* This terminology follows Varga [2].
} 
but, since strict inequality holds in (28) for at least one $i$, Theorem 3 of [3] implies $\left|\lambda_{i}\right|<1$, therefore, $\rho(B)=\sup _{i} \lambda_{i}<1$ and $B$ is convergent.

The block Jacobi matrix $B$ as given by

$$
B=I-D_{1}^{-1} C
$$

indicates that $B^{2}$ does not necessarily have real positive eigenvalues, so finding an optimum acceleration parameter for successive overrelaxation is not an easy matter. However, the following relationship between the eigenvalues $\lambda$ of the successive overrelaxation matrix and the eigenvalues $\mu$ of the block Jacobi matrix still holds, i.e.,

$$
(\lambda+\omega-1)^{2}=\lambda \mu^{2} \omega^{2} .
$$

This implies that the Gauss-Seidel method (i.e., $\omega=1$ ) has twice the asymptotic rate of convergence as the Jacobi method and also indicates, by continuity, that for $\omega$ near 1 the successive overrelaxation method will be convergent. Therefore experimenting with $\omega$ 's not equal to 1 is recommended.

Conclusions. The problem described by equations (6) and approximated by equations (12) and (13) permits an interesting practical application of block diagonally dominant matrices. Using this concept, we have proved convergence of certain iterative methods for solving the system of simultaneous equations of (18). Successive overrelaxation can be rigorously applied to solve the system of equations (18); however, no estimates of convergence rates were obtained. The extensions of this work to large block methods and the finding of an optimum acceleration parameter are still open problems.

Gulf Research and Development Company

Pittsburgh, Pennsylvania

1. A. S. DeWitTe \& D. Lowitz, "Theory of the induction log," Proceedings of the Society of Professional Well Log Analysts: Second Annual Logging Symposium, May 18-19, 1961.

2. R. S. VARGA, Matrix Iterative Analysis, Prentice-Hall, Englewood Cliffs, N. J., 1962. MR $28 * 1725$.

3. R. S. FeINGold \& R. S. VARGA, "Block diagonally dominant matrices and generalizations of the Gerschgorin circle theorem," Pacific J. Math., v. 12, 1962, pp. 1241-1250, MR 27 * 1458 .

4. M. Fiedler \& V. P'́AK, "Some inequalities for the spectrum of a matrix," Mat. Fyz. Časopis Sloven. Akad. Vied, v. 10, 1960, pp. 148-166.

5. A. M. Ostrowski, "On some metrical properties of operator matrices and matrices partitioned into blocks," J. Math. A nal. Appl., v. 2, 1961,pp. 161-209. MR 24 * A421.

6. J. A. Strat'Ton, Electromagnetic Theory, McGraw-Hill, New York, 1941.

7. J. 1). JAckson, C'lassical Electrodynamics, Wiley, New York, 1962. 